\title{
Nonlinear Coherent Modes of Trapped Bose-Einstein Condensates
}

\author{
V.I. Yukalov ${ }^{1,2}$, E.P. Yukalova ${ }^{1,3}$, and V.S. Bagnato ${ }^{1}$ \\ ${ }^{1}$ Instituto de Fisica de São Carlos, Universidade de São Paulo \\ Caixa Postal 369, São Carlos, São Paulo 13560-970, Brazil \\ ${ }^{2}$ Bogolubov Laboratory of Theoretical Physics \\ Joint Institute for Nuclear Research, Dubna 141980, Russia \\ ${ }^{3}$ Department of Computational Physics, Laboratory of Information Technologies \\ Joint Institute for Nuclear Research, Dubna 141980, Russia
}

Nonlinear coherent modes are the collective states of trapped Bose atoms, corresponding to different energy levels. These modes can be created starting from the ground state condensate that can be excited by means of a resonant alternating field. A thorough theory for the resonant excitation of the coherent modes is presented. The necessary and sufficient conditions for the feasibility of this process are found. Temporal behaviour of fractional populations and of relative phases exhibits dynamic critical phenomena on a critical line of the parametric manifold. The origin of these critical phenomena is elucidated by analyzing the structure of the phase space. An atomic cloud, containing the coherent modes, possesses several interesting features, such as interference patterns, interference current, spin squeezing, and massive entanglement. The developed theory suggests a generalization of resonant effects in optics to nonlinear systems of Bose-condensed atoms.

03.75.-b, 03.75. Fi 


\section{INTRODUCTION}

Low-temperature properties of dilute Bose-Einstein condensate are well described by the Gross-Pitaevskii equation (see reviews [1-3]). The mathematical structure of the latter equation is that of the nonlinear Schrödinger equation. This equation in the presence of a confining potential possesses a discrete spectrum of stationary states. The groundstate solution corresponds to the equilibrium Bose condensate. If it could be possible to macroscopically populate a nonground-state energy level, this would correspond to the creation of a nonground-state Bose-Einstein condensate. The feasibility of creating such nonground-state condensates was advanced in Ref. [4]. The properties of these excited self-consistent states and different ways of their formation have been previously discussed in [4-12]. A nonlinear dipole mode in a two-component condensate was excited in experiment [13].

A few words are in order to clarify the meaning of the self-consistent excited states. First of all, they should not be confused with the elementary collective excitations. The latter correspond to small oscillations around a given trap state, say around the ground state, and are described by the linear Bogolubov-De Gennes equations. While the self-consistent trap states are described by the nonlinear Gross-Pitaevskii equation. This is why the stationary solutions to the latter equation are called the nonlinear modes. The Bose-condensed atoms possess a high degree of coherence [1-3], and the related nonlinear Schrödinger equation is known to describe a coherent wave function, or the coherent field [14]. Because of this, the nonlinear trap modes are termed the coherent modes. The atomic densities, corresponding to different nonlinear coherent modes, possess rather different spatial distribution, because of which such modes are sometimes named as topological [3,13]. Vortices are an example of these modes. The time-dependent nonlinear Schrödinger equation supports the existence of solutions called solitons $[15,16]$. The latter are certainly nonlinear by their origin, but they are not necessarily identical to the nonlinear coherent modes. Only those solitons can be identified with such modes, which are the solutions to the stationary eigenvalue problem.

In the present paper, we study the excitation of the nonlinear coherent modes by means of the resonance pumping technique suggested in Ref. [4]. To some extent, this is analogous to the problem of periodically modulated nonlinear optical fibers [17], as opposed to the adiabatic perturbations of nonlinear soliton equations [18]. Our aim here is to develop a thorough theory of the nonlinear coherent modes, analyzing their features that have not been considered in our previous publications $[4,7]$. The main new points are as follows:

(1) A more general pumping field is considered, which makes it easier to create vortices and, what is more important, allows the variation of the initial phase difference related to the resonantly connected fractional populations. Varying this phase difference makes the dynamical picture much richer, providing several novel regimes of motion.

(2) Necessary and sufficient conditions for the feasibility of the resonant excitation of the nonlinear modes are derived. These conditions impose limitations on the admissible strength of atomic interactions and on the number of trapped atoms. The found restriction on the number of atoms is similar to the critical number arising in the analysis of the stability of condensates with a negative scattering length. The ways of optimizing the found limits are discussed.

(3) Temporal dynamics of fractional populations and of the related phase difference is studied in detail for all admissible initial conditions and for varying system parameters. Stability analysis is accomplished. Investigating the phase portrait determines possible dynamic phase transitions.

(4) A special attention is paid to dynamic critical phenomena, discovered earlier $[19,20]$ by means of numerical calculations, but which have not received a comprehensive explanation. The origin of these critical phenomena is elucidated as being due to the saddle separatrix crossing by the starting point of a trajectory in phase space. A set of critical lines on the parametric manifold is found.

(5) The influence of power broadening on the long lasting resonant pumping is considered. This effect, despite well preserved resonance conditions on the excitation frequency, may lead to the accumulation of perturbations, resulting in the appearance of attenuation, limiting the admissible time of pumping. This limiting time is found to be of order of the trapping lifetime caused by depolarizing atomic collisions.

(6) Interference pattern and interference current are found to display two-scale oscillations, slow and fast. These interference effects can be experimentally observed.

(7) Atomic spin squeezing in the presence of multiparticle entanglement is studied. The dispersion of the populationdifference operator is always squeezed with respect to that of the transition-dipole operator. This means that the fractional populations can be measured with a precision that is higher than the accuracy of measuring transitional characteristics, such as the relative phase. The connection of these results with the notions of state coherence and transition coherence is discussed. 


\section{COHERENT MODES}

The Gross-Pitaevskii equation is usually treated as an approximate mean-field equation for the broken symmetry order parameter $[1,2]$. This interpretation, however, is not unique. Here we show that this equation can be considered as an exact equation for a coherent wave function. The latter interpretation sheds some light on the intimate relation between Bose-Einstein condensate and coherence. At the same time, this allows us to give a rigorous mathematical definition of coherent modes which will be considered in the following sections. To suggest an accurate definition of the nonlinear coherent modes is of principal importance, since these are the basic object studied in the present paper.

In the field approach, dealing with the field operators $\psi(\mathbf{r}, t)$, the standard multiatomic Hamiltonian reads

$$
\begin{gathered}
H=\int \psi^{\dagger}(\mathbf{r}, t)\left[-\frac{\hbar^{2} \nabla^{2}}{2 m_{0}}+U(\mathbf{r}, t)\right] \psi(\mathbf{r}, t) d \mathbf{r}+ \\
+\frac{1}{2} \int \psi^{\dagger}(\mathbf{r}, t) \psi^{\dagger}\left(\mathbf{r}^{\prime}, t\right) \Phi\left(\mathbf{r}-\mathbf{r}^{\prime}\right) \psi\left(\mathbf{r}^{\prime}, t\right) \psi(\mathbf{r}, t) d \mathbf{r} d \mathbf{r}^{\prime}
\end{gathered}
$$

where $m_{0}$ is atomic mass; $U(\mathbf{r}, t)$, external potential; and $\Phi(\mathbf{r})$, interaction potential. The field operators satisfy the Bose commutation relations. Trapped atoms constitute a dilute gas, for which the interaction potential can be presented in the Fermi form

$$
\Phi(\mathbf{r})=A_{s} \delta(\mathbf{r}), \quad A_{s} \equiv 4 \pi \hbar^{2} \frac{a_{s}}{m_{0}}
$$

in which $a_{s}$ is an $s$-wave scattering length.

Coherent states [21] are defined as the eigenstates of the annihilation operator

$$
\psi(\mathbf{r}, t)|\eta\rangle=\eta(\mathbf{r}, t)|\eta\rangle
$$

Here $|\eta\rangle$ is a coherent state and the function $\eta(\mathbf{r}, t)$, playing the role of an eigenvalue, is called the coherent wave function, or, for brevity, the coherent field. The Heisenberg equation of motion for the field operator yields

$$
i \hbar \frac{\partial}{\partial t} \psi(\mathbf{r}, t)=H[\psi] \psi(\mathbf{r}, t)
$$

with the nonlinear Hamiltonian

$$
H[\psi] \equiv-\frac{\hbar^{2} \nabla^{2}}{2 m_{0}}+U(\mathbf{r}, t)+A_{s} \psi^{\dagger}(\mathbf{r}, t) \psi(\mathbf{r}, t)
$$

Taking the average of Eq. (4) over the coherent state $|\eta\rangle$ gives the equation

$$
i \hbar \frac{\partial}{\partial t} \eta(\mathbf{r}, t)=H[\eta] \eta(\mathbf{r}, t)
$$

for the coherent field, with the nonlinear Hamiltonian

$$
H[\eta] \equiv-\frac{\hbar^{2} \nabla^{2}}{2 m_{0}}+U(\mathbf{r}, t)+A_{s}|\eta(\mathbf{r}, t)|^{2} .
$$

At zero temperature, when all $N$ atoms are condensed into a coherent state, the coherent field $\eta(\mathbf{r}, t)$ is normalized to $N$. It is convenient to define

$$
\eta(\mathbf{r}, t) \equiv \sqrt{N} \varphi(\mathbf{r}, t)
$$

The external potential

$$
U(\mathbf{r}, t)=U(\mathbf{r})+V(\mathbf{r}, t)
$$

consists of a stationary trapping potential $U(\mathbf{r})$ and of an additional perturbing potential $V(\mathbf{r}, t)$. Then Eq. (6) for the coherent field can be written as 


$$
i \hbar \frac{\partial}{\partial t} \varphi(\mathbf{r}, t)=(\hat{H}[\varphi]+\hat{V}) \varphi(\mathbf{r}, t)
$$

where $\hat{V} \equiv V(\mathbf{r}, t)$ and

$$
\hat{H}[\varphi] \equiv-\frac{\hbar^{2} \nabla^{2}}{2 m_{0}}+U(\mathbf{r})+N A_{s}|\varphi(\mathbf{r}, t)|^{2} .
$$

Equations (6) and (10) are exact equations for the coherent fields. Such equations in physical literature are termed the time-dependent Gross-Pitaevskii equations. In mathematical parlance, these are the nonlinear Schrödinger equations $[15,16]$. Being complimented by initial and boundary conditions, such equations compose the Cauchy problem. For nonlinear differential equations in partial derivatives, the Cauchy problem possesses a unique analytic solution under the conditions of the Cauchy-Kovalevskaya theorem [22]. These conditions are not fulfilled for Eqs. (6) or (10). Hence these equations may, in general, have several solutions. To guarantee the uniqueness of solution, it is necessary to impose some additional constraints motivated by the physics of the problem $[15,16]$. In particular, it is necessary to specify a functional space to which the sought solution is supposed to pertain. For the studied equations, it is possible to proceed as follows.

Let us consider the eigenproblem

$$
\hat{H}\left[\varphi_{n}\right] \varphi_{n}(\mathbf{r})=E_{n} \varphi_{n}(\mathbf{r}),
$$

defining the stationary solutions $\varphi_{n}(\mathbf{r})$ labelled by a multi-index $n$. In the presence of a confining potential $U(\mathbf{r})$, the spectrum $\left\{E_{n}\right\}$ is discrete. The countability of the set $\left\{\varphi_{n}(\mathbf{r})\right\}$ for a nonlinear problem is not absolutely guaranteed. For the Gross-Pitaevskii equation with a single-well confining potential, the eigenproblem can be treated as an analytical continuation of that for the associated linear Schrödinger equation resulting in the limit of vanishing nonlinearity $[10,12]$. Then the set $\left\{\varphi_{n}(\mathbf{r})\right\}$ is easily countable. But in general, e.g. in the case of multiwell confining potentials, there appear the stationary solutions to the Gross-Pitaevskii equation, which have no linear counterparts [12], because of which the countability problem becomes rather nontrivial. However, for what follows, the countability of the set of stationary solutions to Eq. (12) is not compulsory. The main is that these solutions be appropriately labelled by a multi-index. The solutions $\varphi_{n}(\mathbf{r})$ to the nonlinear eigenproblem (12) are the nonlinear coherent modes. Generally, some of the levels can be degenerate, which does not hamper the consideration. Moreover, the problem of degeneracy, when it becomes annoying, can be avoided by adding to the Hamiltonian $\hat{H}\left[\varphi_{n}\right]$ terms lifting the degeneracy. Such auxilliary terms may be removed after accomplishing the required procedures. The main is that the set $\left\{\varphi_{n}(\mathbf{r})\right\}$ be composed of all possible different solutions. This, however, does not presuppose that all functions from the set are mutually orthogonal, so that the scalar product

$$
\left(\varphi_{m}, \varphi_{n}\right) \equiv \int \varphi_{m}^{*}(\mathbf{r}) \varphi_{n}(\mathbf{r}) d \mathbf{r}
$$

is not compulsory a Kroneker delta. The possible nonorthogonality of the coherent modes may be due to the fact that the Hamiltonian $\hat{H}[\varphi]$ is nonlinear. In such a case, one has

$$
\left(\hat{H}\left[\varphi_{m}\right] \varphi_{m}, \varphi_{n}\right)-\left(\varphi_{m}, \hat{H}\left[\varphi_{n}\right] \varphi_{n}\right)=\left(E_{m}-E_{n}\right)\left(\varphi_{m}, \varphi_{n}\right) .
$$

The left-hand side of the latter equality, for the nonlinear Hamiltonian (11), reads

$$
\left(\hat{H}\left[\varphi_{m}\right] \varphi_{m}, \varphi_{n}\right)-\left(\varphi_{m}, \hat{H}\left[\varphi_{n}\right] \varphi_{n}\right)=N A_{s}\left(\varphi_{m},\left[\left|\varphi_{m}\right|^{2}-\left|\varphi_{n}\right|^{2}\right] \varphi_{n}\right),
$$

which, in general, is not zero. Therefore, the modes $\varphi_{m}$ and $\varphi_{n}$, corresponding to $E_{m} \neq E_{n}$, are not necessarily orthogonal. But the modes can, of course, be always normalized, so that $\left(\varphi_{n}, \varphi_{n}\right)=1$. Now, let us organize a closed linear envelope over the set $\left\{\varphi_{n}(\mathbf{r})\right\}$, which, being equipped with the scalar product (13), composes a Hilbert space

$$
\mathcal{H} \equiv \overline{\mathcal{L}}\left\{\varphi_{n}(\mathbf{r})\right\} .
$$

By this construction, the set $\left\{\varphi_{n}(\mathbf{r})\right\}$ is total [21], which implies that a function from the Hilbert space (14) can be presented as an expansion over this set. For instance, looking for a solution of Eq. (10), pertaining to the space (14), we may write the mode presentation

$$
\varphi(\mathbf{r}, t)=\sum_{n} b_{n}(t) \varphi_{n}(\mathbf{r})
$$


One should not confuse the property of totality with that of completeness. The totality of a set $\left\{\varphi_{n}(\mathbf{r})\right\}$ means the possibility of presenting a function $\varphi \in \mathcal{H}$ from the Hilbert space (14) as the sum (15). While the completeness of a set $\left\{\varphi_{n}(\mathbf{r})\right\}$ implies the existence of the resolution of unity

$$
\sum_{n} \rho_{n} \varphi_{n}(\mathbf{r}) \varphi_{n}^{*}\left(\mathbf{r}^{\prime}\right)=\delta\left(\mathbf{r}-\mathbf{r}^{\prime}\right),
$$

in general, involving a weighting set $\left\{\rho_{n}\right\}$. More details on the delicate difference between totality and completeness can be found in the book [21].

The property of totality is weaker than that of completeness. A complete basis is certainly total, while a total set is not compulsory complete. In our case, the set $\left\{\varphi_{n}(\mathbf{r})\right\}$ of coherent modes, defined by the eigenproblem (12), may happen to be complete. A hint to this is the observation [10] that the eigenstates of the nonlinear problem (12) can be considered as an analytical continuation, with respect to the nonlinearity constant $A_{s}$, of the eigenstates for the related linear problem, which possesses a complete basis. Moreover, the completeness of the countable set of eigenfunctions to the one-dimensional nonlinear Schrödinger Hamiltonian has been rigorously proved [23,24]. There is no such an exact proof of completeness for the three-dimensional case. But we would like to stress that nowhere in what follows we shall use the completeness of the set $\left\{\varphi_{n}(\mathbf{r})\right\}$ and will never invoke the related resolution of unity. The sole thing we shall employ is the totality of the set of coherent modes, which is equivalent to the presentation (15). Note that for a homogeneous gas, the modes $\varphi_{n}(\mathbf{r})$ reduce to plane waves [25].

\section{RESONANT EXCITATION}

In the case of a discrete spectrum, the energy levels are separated, and the interlevel distance is characterized by the transition frequency

$$
\omega_{m n} \equiv \frac{1}{\hbar}\left(E_{m}-E_{n}\right) .
$$

In order to single out a pair of levels, it is necessary to connect them by a resonant transition. For this, an external oscillatory field

$$
V(\mathbf{r}, t)=V_{1}(\mathbf{r}) \cos \omega t+V_{2}(\mathbf{r}) \sin \omega t
$$

has to be imposed on the atomic system, with a frequency tuned to the transition frequency between a couple of chosen levels, say the levels with the energies $E_{1}<E_{2}$, so that the transition frequency be

$$
\omega_{21} \equiv \frac{1}{\hbar}\left(E_{2}-E_{1}\right) .
$$

Then the resonance condition reads

$$
\left|\frac{\Delta \omega}{\omega}\right| \ll 1 \quad\left(\Delta \omega \equiv \omega-\omega_{21}\right) .
$$

The resonant excitation of nonlinear modes in Bose-Einstein condensates is rather analogous to resonant two-level transitions in isolated atoms [26]. The difference is that in atoms, one considers single-electron levels, while in trapped Bose condensate, one deals with collective coherent states of many atoms. To separate out a pair of levels, coupled by a resonant transition, it is necessary that the spectrum be nonequidistant. This is the case for electronic spectra in atoms. And, fortunately, this is also true for the spectrum of coherent modes of trapped Bose condensates, where the spectrum becomes nonequidistant due to atomic interactions [3,4]. It may happen, that the transition frequencies (16), though being different for $E_{m} \neq E_{n}$, are densely packed, as e.g. is the case for highly excited states of an optical atom [26]. Then the resonance condition (19) has to be strengthened, so that the detuning $\Delta \omega$ be smaller than the transition frequencies between the energy levels neighboring the selected levels with the energies $E_{1}$ and $E_{2}$. Such a situation, because of the uncertainty relation $\Delta \omega \cdot \Delta t \sim 1$, would lead to a long time $\Delta t$ required to excite a specific mode from a densely packed spectrum. The same difficulty may arise for Bose-condensed atomic gases, although for the latter one has more possibility of overcoming the problem, as compared to the case of a single atom. This is because a single atom possesses a fixed energy spectrum, while the spectrum of nonlinear coherent modes can be modified by varying the number of particles $N$ or the strength $A_{s}$ of atomic interactions, e.g., by means of Feshbach resonance. At the same time, these interactions render the system nonlinear, which leads to some important 
differences in the behaviour of Bose condensate, subject to the action of a resonant field, as compared to optical resonance in atoms. Comparing these two types of resonance, we may say that in a single atom, one can induce a linear resonance, while in a collective of coherent atoms, there occurs a nonlinear coherent resonance.

The behaviour of the coherent field (15) is described by the nonlinear equation (10), where the alternating field (17) is in resonance with the transition frequency (18) of a chosen interlevel transition. Earlier, we have considered [4,7] only the transitions from the ground state to an excited collective state. But if one is able to transfer atoms from the ground state to an excited level, then by applying one more alternating field, with a different resonant frequency, it is possible to transfer atoms from one excited level to another higher level. Therefore, we consider the general situation of an arbitrary pair of collective levels coupled by a resonant transition.

The coefficients $b_{n}(t)$ in the mode presentation (15) can be written as

$$
b_{n}(t)=c_{n}(t) \exp \left(-\frac{i}{\hbar} E_{n} t\right),
$$

with $E_{n}$ being the $n$-mode energy. In order that different modes could be separated, it is necessary that the coefficient $c_{n}(t)$ be a slow function of time, as compared to the fast oscillations of the exponential $\exp \left(-\frac{i}{\hbar} E_{n} t\right)$. This implies the validity of the necessary condition for mode separation

$$
\frac{\hbar}{E_{n}}\left|\frac{d c_{n}}{d t}\right| \ll 1
$$

Inequality (21) reminds us the slowly-varying amplitude approximation, so common in optics [26]. Under this condition, we may try to get a nontrivial mode picture, following the way accepted in optical resonance. For this purpose, we substitute the mode form (15) into the evolution equation (10), multiply the latter by $\varphi_{n}^{*}(\mathbf{r}) \exp \left(\frac{i}{\hbar} E_{n} t\right)$, integrate it over $\mathbf{r}$, and average over time, treating $c_{n}(t)$ as quasi-invariants. The mathematical foundation of this procedure is based on the averaging technique [27]. In this way, we meet two transition amplitudes, one that is caused by atomic interactions

$$
\alpha_{m n} \equiv A_{s} \frac{N}{\hbar} \int\left|\varphi_{m}(\mathbf{r})\right|^{2}\left[2\left|\varphi_{n}(\mathbf{r})\right|^{2}-\left|\varphi_{m}(\mathbf{r})\right|^{2}\right] d \mathbf{r},
$$

and another due to the alternating field,

$$
\beta_{m n} \equiv \frac{1}{\hbar} \int \varphi_{m}^{*}(\mathbf{r})\left[V_{1}(\mathbf{r})-i V_{2}(\mathbf{r})\right] \varphi_{n}(\mathbf{r}) d \mathbf{r} .
$$

Recall that in optics one has only the transition amplitude of an external resonant field defining a Rabi frequency. Here, because of atomic interactions, there appears an additional amplitude (22). Note that for different energy levels, the amplitude (22) may have different signs, for a fixed sign of the interaction parameter $A_{s}$.

The condition for mode separation (21) is necessary, however not sufficient. For employing the averaging technique [27], we need that the transition amplitudes be smaller than the related transition frequencies,

$$
\left|\frac{\alpha_{m n}}{\omega_{m n}}\right| \ll 1, \quad\left|\frac{\beta_{m n}}{\omega_{m n}}\right| \ll 1 .
$$

This mathematical requirement for the applicability of the averaging technique has a transparent physical meaning all perturbations, either produced by internal interactions or by external fields, must be smaller than the corresponding transition frequencies, in order that the latter be well defined as such. Again, the situation here is completely analogous to optics, where one requires that the Rabi frequencies be smaller than the transition frequency, in order to avoid the power broadening spoiling the resonance picture. In our case, the difference with optics is that we have, in addition to the external transition amplitude (23), an internal amplitude (22). The latter appears because of the interaction term in the nonlinear Hamiltonian (11). This term depends on time through the function $\varphi(\mathbf{r}, t)$. Therefore, one could naively decide that as soon as the coherent field (15) changes from one mode to another, the resonance condition would be broken. However, this does not happen if the restrictions (24) are hold true. Then, despite the variation of the interaction term, the transition frequencies are always well defined, making it possible to tune a well preserved resonance.

Restrictions (24) are stronger than inequality (21). The latter is only necessary, while the former are necessary and sufficient for mode separation. Mathematically, conditions (24) allow us, when employing the averaging technique, to use the integral

$$
\lim _{T \rightarrow \infty} \frac{1}{T} \int_{0}^{T} \exp \left(i \omega_{m n} t\right) d t=\delta_{m n}
$$


As a result, we come to the set of equations

$$
i \frac{d c_{n}}{d t}=\sum_{m(\neq n)} \alpha_{m n}\left|c_{m}\right|^{2} c_{n}+\frac{1}{2} \delta_{n 1} \beta_{12} c_{2} e^{i \Delta \omega t}+\frac{1}{2} \delta_{n 2} \beta_{12}^{*} c_{1} e^{-i \Delta \omega t},
$$

for the functions $c_{n}=c_{n}(t)$, satisfying the normalization condition

$$
\sum_{n}\left|c_{n}\right|^{2}=1
$$

From Eq. (25) it immediately follows that, if $n \neq 1,2$, then one has

$$
c_{n}(t)=c_{n}(0) \exp \left\{-i \sum_{m(\neq n)} \alpha_{n m} \int_{0}^{t}\left|c_{m}\left(t^{\prime}\right)\right|^{2} d t^{\prime}\right\} .
$$

Supposing that at the initial time no levels, except $n=1,2$, are populated, that is $c_{n}(0)=0$ for $n \neq 1,2$, we get from Eq. (26) that

$$
c_{n}(t)=0 \quad(n \neq 1,2 ; t \geq 0) .
$$

Hence, the normalization condition is

$$
\left|c_{1}\right|^{2}+\left|c_{2}\right|^{2}=1
$$

And the set of equations (25) reduces to two equations

$$
i \frac{d c_{1}}{d t}=\alpha_{12}\left|c_{2}\right|^{2} c_{1}+\frac{1}{2} \beta_{12} c_{2} e^{i \Delta \omega t}, \quad i \frac{d c_{2}}{d t}=\alpha_{21}\left|c_{1}\right|^{2} c_{2}+\frac{1}{2} \beta_{12}^{*} c_{1} e^{-i \Delta \omega t} .
$$

This reduction is valid provided that the conditions

$$
\left|\frac{\alpha_{12}}{\omega_{21}}\right| \ll 1, \quad\left|\frac{\alpha_{21}}{\omega_{21}}\right| \ll 1, \quad\left|\frac{\beta_{12}}{\omega_{21}}\right| \ll 1
$$

are satisfied.

It would be tempting to postulate from the very beginning that, under the resonance condition (19), only two modes are involved in the process. However such a simplified approach would leave doubts of the possibility to reduce the consideration to an effective two-level system. Therefore, we felt it necessary to present an accurate mathematical derivation of the reduced equations (29). Moreover, in the process of this derivation, we found conditions (30) defining the region of applicability for the resonant two-level picture.

By acting on the trapped Bose gas with several resonant fields, we could separate out not two but several coherent modes, reducing the consideration to a finite-level system. The possibility of creating from Bose condensate the resonant finite-level systems is of great importance. This allows for a wide variety of different applications, analogous to those for resonant finite-level atoms in optics $[26,28]$. Since the properties of resonant Bose-condensed systems are rather different from those of single resonant atoms, we may expect the appearance of novel effects, such as dynamic critical phenomena $[19,20]$. In what follows, for brevity, the effect of resonant formation of nonlinear coherent modes in Bose-condensed gas will be called coherent resonance.

Note that a resonant finite-level system of coherent modes is principally different from the two-mode models accepted for describing Bose condensates in stationary double-well potentials [8,29-30]. Though some equations in our case may resemble some expressions in the case of the double-well potentials, this similarity is rather formal, being due to the simple fact that all finite-level systems share some common properties in their mathematical structure. However the physics of a system, subject to the action of a resonant alternating field, drastically differs from what happens in stationary double wells.

\section{TRANSITION AMPLITUDES}

The set of inequalities (19) and (30) provides us with the necessary and sufficient conditions for realizing the coherent resonance. The frequency and the amplitude of the alternating external field (17) can always be chosen in order to 
satisfy the resonance condition (19) and the last of the inequalities (30). Hence the main concern is connected with the validity of the first two inequalities (30) for the internal transition amplitudes (22) due to atomic interactions. To understand better what is the restriction imposed by these inequalities, let us consider a harmonic cylindrical trap with the radial frequency $\omega_{r} \equiv \omega_{x}=\omega_{y}$ and axial frequency $\omega_{z}$, denoting the aspect ratio

$$
\nu \equiv \frac{\omega_{z}}{\omega_{r}}
$$

Introduce the dimensionless cylindrical variables

$$
r \equiv \frac{\sqrt{r_{x}^{2}+r_{y}^{2}}}{l_{r}}, \quad z \equiv \frac{r_{z}}{l_{r}} \quad\left(l_{r} \equiv \sqrt{\frac{\hbar}{m_{0} \omega_{r}}}\right),
$$

measured in units of the oscillator length $l_{r}$. In the dimensionless coupling parameter

$$
g \equiv 4 \pi \frac{a_{s}}{l_{r}} N,
$$

$a_{s}$ is an $s$-wave scattering length and $N$ is the number of particles. The nonlinear Hamiltonian, measured in units of $\omega_{r}$, is

$$
\hat{H}=-\frac{1}{2} \nabla^{2}+\frac{1}{2}\left(r^{2}+\nu^{2} z^{2}\right)+g|\psi|^{2},
$$

where

$$
\psi(r, \varphi, z) \equiv l_{r}^{3 / 2} \varphi(\mathbf{r})
$$

is a dimensionless wave function and

$$
\nabla^{2}=\frac{\partial^{2}}{\partial r^{2}}+\frac{1}{r} \frac{\partial}{\partial r}+\frac{1}{r^{2}} \frac{\partial^{2}}{\partial \varphi^{2}}+\frac{\partial^{2}}{\partial z^{2}}
$$

In this notation, the eigenproblem (12) reads

$$
\hat{H} \psi_{n m j}(r, \varphi, z)=E_{n m j} \psi_{n m j}(r, \varphi, z),
$$

where $n=0,1,2, \ldots$ is the radial quantum number, $m=0, \pm 1, \pm 2, \ldots$ is the azimuthal quantum number, and $j=0,1,2, \ldots$ is the axial quantum number.

The eigenproblem (35) can be solved by employing the optimized perturbation theory [33-35]. For this purpose, we take as an initial approximation the Hamiltonian

$$
\hat{H}_{0}=-\frac{1}{2} \nabla^{2}+\frac{1}{2}\left(u^{2} r^{2}+v^{2} z^{2}\right),
$$

containing two trial parameters, $u$ and $v$, playing the role of effective dimensionless frequencies. This Hamiltonian possesses the eigenfunctions

$$
\begin{gathered}
\psi_{n m j}^{0}(r, \varphi, z)=\left[\frac{2 n ! u^{|m|+1}}{(n+|m|) !}\right]^{1 / 2} r^{|m|} \exp \left(-\frac{u}{2} r^{2}\right) L_{n}^{|m|}\left(u r^{2}\right) \times \\
\times \frac{e^{i m \varphi}}{\sqrt{2 \pi}}\left(\frac{v}{\pi}\right)^{1 / 4} \frac{1}{\sqrt{2^{j} j !}} \exp \left(-\frac{v}{2} z^{2}\right) H_{j}(\sqrt{v} z),
\end{gathered}
$$

where $L_{n}^{m}(\cdot)$ is a Laguerre polynomial and $H_{j}(\cdot)$ is a Hermit polynomial. Then, invoking the Rayleigh-Schrödinger perturbation theory, we may find the higher approximations. Thus, for the spectrum, we get the sequence $\left\{E_{k}(g, u, v)\right\}$ of approximations, with $k=0,1,2, \ldots$ being an approximation order. For instance, in the first order,

$$
E_{1}(g, u, v)=\frac{p}{2}\left(u+\frac{1}{u}\right)+\frac{q}{4}\left(v+\frac{\nu^{2}}{v}\right)+u \sqrt{v} g I_{n m j},
$$


where the dependence of the energy on the quantum numbers is not explicitly marked in order to avoid cumbersome notation; the quantum numbers enter through the combinations

$$
p \equiv 2 n+|m|+1, \quad q \equiv 2 j+1
$$

and through the integral

$$
I_{n m j} \equiv \frac{1}{u \sqrt{v}} \int\left|\psi_{n m j}(r, \varphi, z)\right|^{4} r d r d \varphi d z
$$

Then the trial parameters $u$ and $v$ are to be transformed to control functions $u_{k}(g)$ and $v_{k}(g)$, which play here the role of control frequencies, such that the sequence $\left\{E_{k}\left(g, u_{k}(g), v_{k}(g)\right)\right\}$ be convergent [33]. This transformation is accomplished by means of an optimization condition. Limiting ourselves by the first-order approximation, we may write the optimization condition as

$$
\left(\delta u \frac{\partial}{\partial u}+\delta v \frac{\partial}{\partial v}\right) E_{1}(g, u, v)=0 .
$$

In view of expression (37), this gives the equations

$$
p\left(1-\frac{1}{u^{2}}\right)+\frac{G}{p \nu} \sqrt{\frac{v}{q}}=0, \quad q\left(1-\frac{\nu^{2}}{v^{2}}\right)+\frac{u G}{p \nu \sqrt{v q}}=0
$$

for the control frequencies $u=u(g)$ and $v=v(g)$, where the notation

$$
G \equiv 2 p \sqrt{q} I_{n m j} g \nu
$$

is used. Substituting these control functions into Eq. (37), we obtain the optimized approximant

$$
E(g) \equiv E_{1}(g, u(g), v(g))
$$

for the energy spectrum. Recall that the energy (41) as well as the control frequencies $u=u_{m n j}$ and $v=v_{n m j}$ depend on quantum numbers.

The spectrum (41), for arbitrary values of $g$, is defined by the system of equations (37) and (39). In the limits of weak coupling and strong coupling, we can derive explicit asymptotic expansions. Thus, for weak coupling, when $g \rightarrow 0$ and $G \rightarrow 0$, the control frequencies are

$$
u \simeq 1-\frac{G}{2 p^{2}(q \nu)^{1 / 2}}, \quad v \simeq \nu-\frac{G \nu}{2 p(q \nu)^{3 / 2}} .
$$

And the energy spectrum is

$$
E \simeq a_{0}+a_{1} G \quad(G \rightarrow 0)
$$

where

$$
a_{0}=p+\frac{q \nu}{2}, \quad a_{1}=\frac{1}{2 p(q \nu)^{1 / 2}} .
$$

In the limit of strong coupling, the control frequencies are

$$
u \simeq \frac{p}{G^{2 / 5}}, \quad v \simeq \frac{q \nu^{2}}{G^{2 / 5}} .
$$

And for the energies, we find

$$
E \simeq b_{0} G^{2 / 5}+b_{1} G^{-2 / 5} \quad(G \rightarrow \infty)
$$

where

$$
b_{0}=\frac{5}{4}, \quad b_{1}=\frac{2 p^{2}+(q \nu)^{2}}{4} .
$$


The difference between two selected energy levels defines the transition frequency (18).

If we consider transitions between the ground-state level $(n=m=j=0)$ and an excited level, then calculating the internal transition amplitudes (22), we meet the integral

$$
J_{n m j} \equiv \frac{1}{u_{0} \sqrt{v_{0}}} \int\left|\psi_{0}(r, \varphi, z)\right|^{2}\left|\psi_{n m j}(r, \varphi, z)\right|^{2} r d r d \varphi d z,
$$

in which $u_{0} \equiv u_{000}(g)$ and $v_{0} \equiv v_{000}(g)$. For the transition amplitudes (22), corresponding to transitions between the ground state and an excited mode, labelled by the quantum numbers $n, m$, and $j$, we obtain

$$
\begin{gathered}
\alpha_{0, n m j}=g u_{0} \sqrt{v_{0}}\left(I_{0}-2 J_{n m j}\right), \\
\alpha_{n m j, 0}=g u_{0} \sqrt{v_{0}}\left(\frac{u_{n m j}}{u_{0}} \sqrt{\frac{v_{n m j}}{v_{0}}} I_{n m j}-2 J_{n m j}\right),
\end{gathered}
$$

where $I_{0} \equiv I_{000}$. The first two inequalities in Eq. (30) for the internal transition amplitudes now read

$$
\left|\frac{\alpha_{0, n m j}}{\omega_{n m j, 0}}\right| \ll 1, \quad\left|\frac{\alpha_{n m j, 0}}{\omega_{0, n m j}}\right| \ll 1 .
$$

Introduce the quantities

$$
\alpha_{n m j} \equiv \frac{1}{2}\left(\alpha_{0, m n j}+\alpha_{m n j, 0}\right), \quad \delta_{n m j} \equiv \frac{1}{2}\left(\alpha_{0, m n j}-\alpha_{m n j, 0}\right) .
$$

Here $\alpha_{n m j}$ is an average transition amplitude, and $\delta_{n m j}$ plays the role of an internal detuning induced by atomic interactions. By direct numerical calculations of Eqs. (45) and (47), we have checked that $\left|\delta_{n m j} / \alpha_{n m j}\right| \leq 0.1$. In appendix, we demonstrate calculations for several first excited modes.

The analysis of inequalities (46) shows that their validity imposes a constraint on the renormalized coupling (40), requiring that the latter be outside the region of convergence of the strong-coupling expansion (43). Explicitly, this yields the condition

$$
|g \nu| \leq \frac{\left[2 p^{2}+(q \nu)^{2}\right]^{5 / 4}}{14 p \sqrt{q} I_{n m j}}
$$

The constraint (48) on the coupling parameter (33) implies that the number of particles in the trap has to be less than the limiting number

$$
N_{0}=\frac{\left[2 p^{2}+(q \nu)^{2}\right]^{5 / 4}}{56 \pi \nu p \sqrt{q} I_{n m j}}\left|\frac{l_{r}}{a_{s}}\right| .
$$

The value of $N_{0}$ depends on the quantum numbers, thus, being different for different modes. For the ground state, Eq. (48) becomes

$$
|g \nu| \leq\left(2+\nu^{2}\right)^{5 / 4}
$$

and, respectively, for the limiting number (49), we get

$$
N_{0}=\sqrt{\frac{\pi}{2}} \frac{\left(2+\nu^{2}\right)^{5 / 4}}{14 \nu}\left|\frac{l_{r}}{a_{s}}\right| .
$$

The limiting number increases for the higher excited modes. Thus, it grows as

$$
N_{0} \sim(2 n+|m|+1)^{3 / 2}, \quad N_{0} \sim(2 j+1)^{2}
$$

according to whether the radial or axial modes are excited. For highly excited modes, one could invoke an optimized expansion in powers of $\hbar$ [36]. The number $N_{0}$ is also very sensitive to the trap shape, depending on the aspect ratio (31). For the disk-shape $(\nu \gg 1)$ and cigar-shape $(\nu \ll 1)$ traps, $N_{0}$ is larger than for a spherical $(\nu=1)$ trap.

It is interesting that the limiting number of particles for an excited coherent mode is close to the critical number of atoms for the stability of a trapped Bose gas with attractive interactions. Hence, such gases can also support 
coherent modes. Examples of atoms with a negative scattering length are ${ }^{7} \mathrm{Li}$ (see review [37]) and ${ }^{85} \mathrm{Rb}$ (see Ref. [38]). The recent developments in utilizing Feshbach resonances have shown the ability to tune the scattering length to almost any desired value, including the ability of changing its sign [3], which makes it possible to add to these two species many other atoms. The critical number of trapped Bose-condensed atoms with attractive interactions has been estimated earlier [4,39-41]. Such trapped atoms form a metastable state, which decays because of quantum tunneling, which however, is very slow [42-44], if the number of atoms is less than the critical number. There was a proposal [45] for stabilizing Bose condensate with attractive interactions by driving a quadrupole collective excitation. Our consideration above suggests that it could, probably, be also possible to stabilize such condensates by transferring them in an excited coherent mode with the help of the coherent resonance.

To estimate the limiting number of particles in a coherent mode, consider a cigar-shape trap, as was used [46] for condensing ${ }^{23} \mathrm{Na}$. With the radial frequency $\omega_{r}=1527 \mathrm{~Hz}$ and axial frequency $\omega_{z}=11 \mathrm{~Hz}$, the aspect ratio is $\nu=0.007$. The scattering length of ${ }^{23} \mathrm{Na}$ is $a_{s}=4.498 \times 10^{-7} \mathrm{~cm}$. Since the oscillator length in this case is $l_{r}=1.345 \times 10^{-4} \mathrm{~cm}$, then $l_{r} / a_{s}=300$. The coupling parameter has to be $g \leq 340$. Therefore, the limiting number $N_{0} \sim 10^{4}$. If one would take a very long cigar-shape trap with the aspect ratio $\nu=0.001$, then $g \leq 2400$ and the limiting number could be as large as $N_{0} \sim 10^{5}$.

\section{PHASE PORTRAIT}

To study the dynamical behaviour of the system under coherent resonance, it is convenient to use the population difference

$$
s \equiv\left|c_{2}\right|^{2}-\left|c_{1}\right|^{2},
$$

which varies in the interval $-1 \leq s \leq 1$. Then, in view of the normalization condition (28), we have

$$
\left|c_{1}\right|^{2}=\frac{1-s}{2}, \quad\left|c_{2}\right|^{2}=\frac{1+s}{2} .
$$

Taking this into account, we may write

$$
c_{1}=\sqrt{\frac{1-s}{2}} \exp \left\{i\left(\pi_{1}+\frac{\Delta \omega}{2} t\right)\right\}, \quad c_{2}=\sqrt{\frac{1+s}{2}} \exp \left\{i\left(\pi_{2}-\frac{\Delta \omega}{2} t\right)\right\},
$$

where $\pi_{1}=\pi_{1}(t)$ and $\pi_{2}=\pi_{2}(t)$ are real-valued phases.

To simplify the notation, we introduce the average interaction amplitude

$$
\alpha \equiv \frac{1}{2}\left(\alpha_{12}+\alpha_{21}\right)
$$

which is real. Noticing that the transition amplitude (23), due to the resonant field, is, in general, complex-valued, we can present it as

$$
\beta_{12}=\beta e^{i \gamma}, \quad \beta \equiv\left|\beta_{12}\right| .
$$

Also, we define the effective detuning

$$
\delta \equiv \Delta \omega+\frac{1}{2}\left(\alpha_{12}-\alpha_{21}\right) .
$$

And, finally, we shall need the phase difference

$$
x \equiv \pi_{2}-\pi_{1}+\gamma,
$$

whose initial value $x_{0}$ can be varied by changing the spatial dependence of the resonant field. With this notation, Eqs. (29) transform to the system of equations for the population difference

$$
\frac{d s}{d t}=-\beta \sqrt{1-s^{2}} \sin x
$$

and for the phase difference 


$$
\frac{d x}{d t}=\alpha s+\frac{\beta s}{\sqrt{1-s^{2}}} \cos x+\delta .
$$

These are the equations that can be written in the Hamiltonian form

$$
\frac{d s}{d t}=-\frac{\partial H}{\partial x}, \quad \frac{d x}{d t}=\frac{\partial H}{\partial s},
$$

with the Hamiltonian

$$
H(s, x)=\frac{\alpha}{2} s^{2}-\beta \sqrt{1-s^{2}} \cos x+\delta s .
$$

Let us consider the stationary solutions to Eqs. (57) and (58) in the rectangle of the variables $-1 \leq s \leq 1$ and $0 \leq x \leq 2 \pi$. These solutions are given by the equations

$$
s^{4}+2 \varepsilon s^{3}-\left(1-b^{2}-\varepsilon^{2}\right) s^{2}-2 \varepsilon s-\varepsilon^{2}=0, \quad \sin x=0,
$$

where the notation

$$
b \equiv \frac{\beta}{\alpha}, \quad \varepsilon \equiv \frac{\delta}{\alpha}
$$

is used. In what follows, for simplifying formulas, the dimensionless detuning $\varepsilon$ will be treated as a small parameter, $|\varepsilon| \ll 1$. As we have checked by direct numerical calculations, the quantity $\left(\alpha_{12}-\alpha_{21}\right) / \alpha$ is small, and in addition, it can always be compensated by $\Delta \omega$, so that $\delta / \alpha$ be as small as required.

The phase-portrait picture depends on the value of the parameter $b$ characterizing the amplitude of the resonant field. This parameter, according to notation (61), can be either positive or negative, depending on the sign of the interaction amplitude $\alpha$. The latter can be negative in the case of attractive interactions. Also, it can become negative, as follows from definition (22), even for repulsive interactions, when the resonantly connected levels are such that

$$
2\left(\left|\varphi_{m}\right|^{2},\left|\varphi_{n}\right|^{2}\right)<\left(\left|\varphi_{m}\right|^{2},\left|\varphi_{m}\right|^{2}\right) .
$$

We calculated numerically the scalar products entering the above inequality and found that, when exciting the lower modes, the amplitude $\alpha$ does not change its sign. For this to occur, the resonantly connected modes must be energetically strongly separated.

When $b^{2} \geq 1$, there are three stationary solutions to Eqs. (57) and (58),

$$
\begin{gathered}
s_{1}^{*}=\frac{\varepsilon}{b}=s_{3}^{*}, \quad x_{1}^{*}=0, \quad x_{3}^{*}=2 \pi, \\
s_{2}^{*}=-\frac{\varepsilon}{b}, \quad x_{2}^{*}=\pi .
\end{gathered}
$$

For $0 \leq b<1$, in addition to the stationary points (62), there appear two other fixed points,

$$
\begin{array}{cc}
s_{4}^{*}=\sqrt{1-b^{2}}+\frac{b^{2} \varepsilon}{1-b^{2}}, & x_{4}^{*}=\pi, \\
s_{5}^{*}=-\sqrt{1-b^{2}}+\frac{b^{2} \varepsilon}{1-b^{2}}, \quad x_{5}^{*}=\pi .
\end{array}
$$

The points (62) and (63) are the solutions to Eq. (60), which are simplified taking account of small detuning $|\varepsilon| \ll 1$. When $-1<b \leq 0$, the phase portrait contains seven fixed points, those given by Eq. (62) plus

$$
\begin{gathered}
s_{4}^{*}=s_{6}^{*}, \quad x_{4}^{*}=0, \quad x_{6}^{*}=2 \pi, \\
s_{5}^{*}=s_{7}^{*}, \quad x_{5}^{*}=0, \quad x_{7}^{*}=2 \pi,
\end{gathered}
$$

where $s_{4}^{*}$ and $s_{5}^{*}$ are the same as in Eq. (63). A qualitative change of a phase portrait is what in dynamical theory called a dynamical phase transition. In our case, as follows from Eqs. (62) to (64), there are such dynamical phase transitions at the values of $b=0$ and $b^{2}=1$. One may notice that the phase portraits for $b>0$ and $b<0$ are similar to each other, just being shifted with respect to $x$ by $\pi$. 
The Jacobian matrix associated with Eqs. (57) and (58) is composed of the elements

$$
\begin{array}{ll}
J_{11}=\frac{b s}{\sqrt{1-s^{2}}} \sin x, & J_{12}=-b \sqrt{1-s^{2}} \cos x, \\
J_{21}=1+\frac{b \cos x}{\left(1-s^{2}\right)^{3 / 2}}, & J_{22}=-\frac{b s}{\sqrt{1-s^{2}}} \sin x .
\end{array}
$$

The eigenvalues of this matrix are given by the equation

$$
J^{2}=\frac{b^{2}}{1-s^{2}}\left(s^{2} \sin ^{2} x-\cos ^{2} x\right)-b \sqrt{1-s^{2}} \cos x .
$$

These eigenvalues are to be evaluated at the fixed points listed above. Then for $b^{2} \geq 1$, we have

$$
J_{1}^{ \pm}= \pm i \sqrt{b(b+1)}=J_{3}^{ \pm}, \quad J_{2}^{ \pm}= \pm i \sqrt{b(b-1)},
$$

which shows that all fixed points (62) are the centers. When $0 \leq b<1$, then

$$
J_{1}^{ \pm}= \pm i \sqrt{b(1+b)}=J_{3}^{ \pm}, \quad J_{2}^{ \pm}= \pm \sqrt{b(1-b)}, \quad J_{4}^{ \pm}= \pm i \sqrt{1-b^{2}}=J_{5}^{ \pm},
$$

from where if follows that the fixed point $\left(s_{2}^{*}, x_{2}^{*}\right)$ is a saddle, while all other points in Eqs. (62) and (63) are the centers. For $-1<b \leq 0$, the fixed points (64) are similar to those (63), being shifted by $\pi$ with respect to $x$. All points remain the centers, except $\left(s_{2}^{*}, x_{2}^{*}\right)$ which is a saddle.

The trajectory, passing through a saddle point, is the saddle separatrix, which separates the phase plane onto qualitatively different regions of motion. In the considered case, the separatrix is given by the equality

$$
H(s, x)=H\left(s_{2}^{*}, x_{2}^{*}\right),
$$

which, in the approximation linear in $\varepsilon$, results in the separatrix equation

$$
\frac{s^{2}}{2}-b \sqrt{1-s^{2}} \cos x+\varepsilon s=b .
$$

This equation defines two separatrices the upper and lower ones. If the initial point $\left(s_{0}, x_{0}\right)$ of a trajectory lies below the lower separatrix, then the variation of $s(t)$ is always limited from above. In the same way, if the starting point of a trajectory is above the upper separatrix, then the change of $s(t)$ is always limited from below by the separatrix. Since varying the parameter $b$ moves the separatrices, which is clear from the separatrix equation (69), the whole phase picture essentially changes. The transformation of the phase portrait with varying the parameter $b$, related to the increase of the transition amplitude due to the resonant field, is illustrated in Fig. 1, where only the most characteristic pictures are presented. The detuning $\varepsilon$ in these figures is set zero. A finite detuning $\varepsilon \neq 0$ makes the pictures slightly asymetric with respect to the line $s=0$, but the overall phase portraits are similar, because of which we do not present them here.

The structure of the phase portraits shows that, at small $b$, there are a number of trajectories always lying in the upper part of the plane if their initial points were above the upper separatrix. In particular, if the upper coherent mode was initially highly populated, and the applied resonant field is weak, the mode will retain its high population. In the extreme case, if at the initial time all atoms are in the upper coherent mode, and there is no external field, so that $s_{0}=1$ and $b=0$, then $s(t)=1$ meaning that atoms remain in the upper mode. Such a setup can be achieved in two ways. For instance, by transferring atoms to the upper level through an intermediate third higher mode. Or, even simpler, just by applying, first, a resonant field sufficient for transferring atoms from the ground state to the chosen coherent mode and then switching off this field. Thus atoms remain locked in this upper mode. This mode-locking effect was described in Ref. [4]. Mathematically, it is analogous to the self-trapping of atoms in one of the wells of a stationary double-well potential $[30-32,47]$. However, the physics of the coherent resonance occurring under the action of a resonant external field is absolutely different. Moreover, the mode-locking effect is not the most interesting thing happening in the process of the resonant excitation of coherent modes.

Varying the amplitude of the resonant field, that is, the parameter $b$, in the dynamic behaviour of fractional populations there appear dynamical critical phenomena [19,20], arising on a critical line of the parametric manifold $\{b, \varepsilon\}$. The qualitative behaviour of fractional populations drastically changes when the critical line is crossed. As was shown $[19,20]$, for a stationary system, obtained by time-averaging the considered dynamical system, there appear 
critical phenomena, typical of phase transitions and occurring on the same critical line, where the dynamical critical phenomena are observed. Thus, we may define a pumping capacity, playing the role of an effective specific heat, and an effective susceptibility, which diverge on the critical line $[19,20]$. The origin of these dynamical critical phenomena has not been completely understood. But now, invoking the analysis of this section, we clearly see what happens.

Suppose, we consider a trajectory starting at the point $\left\{s_{0}, x_{0}\right\}$. Strengthening the applied resonant field means increasing the parameter $b$. But changing $b$ implies moving the separatrix given by Eq. (69). When the separatrix crosses the initial point $\left\{s_{0}, x_{0}\right\}$, the trajectory passes from one region of the phase plane to a qualitatively different region, because of which the dynamical behaviour changes qualitatively. Since, in general, we have two independent parameters, the amplitude $b$ and detuning $\varepsilon$, the effect of separatrix crossing can be due to the variation of any of these parameters. A tiny change of one of these can shift the trajectory to a different part of the phase plane. The condition for the separatrix crossing defines, on the parametric manifold $\{b, \varepsilon\}$, the critical line

$$
\frac{s_{0}^{2}}{2}-b_{c} \sqrt{1-s_{0}^{2}} \cos x_{0}+\varepsilon_{c} s_{0}=\left|b_{c}\right| .
$$

Actually, for each set of initial conditions $\left\{s_{0}, x_{0}\right\}$ there exists its own critical line defined by Eq. (70). For the variety of initial conditions, there is an infinite bunch of critical lines.

For example, let us consider the initial condition $s_{0}=-1, x_{0}=0$, when all atoms at the initial time are in the ground mode. Then the critical line (70) reduces to

$$
\left|b_{c}\right|+\varepsilon_{c}=\frac{1}{2} .
$$

In the case of zero detuning, this defines two points $b_{c}= \pm 0.5$. Assume that our concern is the behaviour of atoms with repulsive interactions, such that $b>0$. And let us set $\varepsilon=0$. The population difference $x(t)$ exhibits dramatic changes when $b$ crosses the critical point $b_{c}=0.5$. This is illustrated in Fig. 2, where the change of $b$ around the critical point $b_{c}$ occurs in the fifth decimal digit, so that the variation of $b$ from Fig. 2(a) to Fig. 2(b) makes $\Delta b=0.00001$. Slightly below the critical point $b_{c}$, the population difference $s(t)$ oscillates between -1 and 0 , while the phase difference $x(t)$ monotonely diminishes. Just above the critical point, the oscillation period of $s(t)$ doubles, as well as the amplitude of $s(t)$, as now $s(t)$ varies between -1 and +1 . The form of $s(t)$ also changes acquiring a specific cusp in the middle of the period. The phase difference $x(t)$, instead of being monotonely diminishing, becomes oscillating.

If we are interested in the behaviour of atoms with $b<0$, say with attractive interactions, then similar dramatic changes happen at the critical point $b_{c}=-0.5$. This is shown in Fig. 3. Again, the change of $b$ around the critical point $b_{c}$ occurs in the fifth decimal digit, as for Fig. 2. After crossing the critical point $b_{c}$, the period and amplitude of $s(t)$ double, and the middle-period cusps appear. The shapes of both $s(t)$ and $x(t)$ essentially change. Note that although these dynamical critical phenomena for $b>0$ and $b<0$ are similar, there is a difference in the behaviour of $x(t)$. For $b<0$, the phase $x(t)$ is an oscillating function below as well as above the critical point. While for $b>0$, the phase difference $x(t)$ below the critical point is a monotonely diminishing function.

In Figs. 2 and 3, time is dimensionless (measured in units of $\alpha^{-1}$ ). The return to dimensional time is accomplished by the substitution $t \rightarrow t / \alpha$. A small detuning $\varepsilon \neq 0$ slightly curves the lines but does not make qualitative change.

\section{POWER BROADENING}

The feasibility of coherent resonance imposes a limitation on the value of the internal transition amplitude, that is, as discussed in Sec. IV, on the admissible number of particles. In addition to the amplitude restriction, there is one more limitation having to do with temporal power broadening. Even if the alternating external field is well tuned to the resonance with a chosen transition frequency, nevertheless, there exist nonresonant transitions to other levels. Though the probability of nonresonant transitions, at each given instant of time, is very small, their influence on the process of resonant excitation can increase with time, becoming essential for long time intervals. To estimate this influence, we return to the evolution equations (29).

It is convenient to introduce the quantity

$$
h \equiv 2 c_{1}^{*} c_{2} e^{i(\Delta \omega t+\gamma)}=\sqrt{1-s^{2}} e^{i x},
$$

playing the role of a ladder variable in optics [26,28]. Here $s=s(t)$ and $x=x(t)$ are the same as in Eqs. (51) and (56). Define the collective frequency

$$
\Omega \equiv \alpha s+\delta
$$


Here and in what follows, we use the notation for $\alpha, \beta$, and $\delta$ as given in Eqs. (53), (54), and (55), respectively. Taking account of nonresonant transitions can be done by including in the evolution equations an inhomogeneous broadening modelled by a random variable [28]. Then, Eqs. (29) can be rewritten in the form of the stochastic equations

$$
\begin{gathered}
\frac{d h}{d t}=i(\Omega+\xi) h+i \beta s, \\
\frac{d s}{d t}=\frac{i}{2} \beta\left(h-h^{*}\right),
\end{gathered}
$$

where $\xi=\xi(t)$ is a stochastic variable modelling nonresonant transitions.

In analysing Eqs. (73) and (74), we assume, for simplicity, that the transition amplitude due to the external field is weaker than that caused by internal atomic interactions,

$$
\left|\frac{\beta}{\alpha}\right| \ll 1
$$

The stochastic variable can be presented as Gaussian white noise [48], with the stochastic averages

$$
\ll \xi(t) \gg=0, \quad \ll \xi(t) \xi\left(t^{\prime}\right) \gg=2 \gamma_{3} \delta\left(t-t^{\prime}\right) .
$$

The noise width can be approximated as

$$
\gamma_{3} \approx \frac{\alpha^{2}+\beta^{2}}{\omega_{21}}
$$

so that, according to inequalities $(30), \gamma_{3} \ll \omega_{21}$.

Equations (73) and (74) can be solved by employing the scale separation approach [49-51], which is a generalization of the averaging technique $[27,52]$ to stochastic differential equations. Because of inequality $(75)$, the function $h=h(t)$ can be treated as fast, compared to the slow function $s=s(t)$. The latter is a temporal quasi-invariant with respect to $h$. In this case, the solution to Eq. (73) reads

$$
h=h_{0} e^{i \Omega t} \exp \left\{i \int_{0}^{t} \xi\left(t^{\prime}\right) d t^{\prime}\right\}+i \beta s \int_{0}^{t} e^{i \Omega\left(t-t^{\prime}\right)} \exp \left\{i \int_{t^{\prime}}^{t} \xi\left(t^{\prime \prime}\right) d t^{\prime \prime}\right\} d t^{\prime},
$$

where $h_{0} \equiv h(0)$. Averaging Eq. (78) over the stochastic variable, with the use of Eqs. (76), we have

$$
\ll h \gg=\left(h_{0}+\frac{\beta s}{\Omega+i \gamma_{3}}\right) e^{\left(i \Omega-\gamma_{3}\right) t}-\frac{\beta s}{\Omega+i \gamma_{3}} .
$$

Substituting the solution (78) for the fast function into Eq. (74) for the slow function and averaging the right-hand side of Eq. (74) over the stochastic variable and over time, we obtain the equation

$$
\frac{d s}{d t}=-\frac{\beta^{2} \gamma_{3} s}{(\alpha s+\delta)^{2}+\gamma_{3}^{2}}
$$

for the guiding center of the slow function. An approximate solution to this equation can be written as

$$
s \approx s_{0} \exp \left(-\frac{\beta^{2} \gamma_{3} t}{\Omega^{2}+\gamma_{3}^{2}}\right) .
$$

This shows that the population difference (81) attenuates, with the characteristic time

$$
t_{c} \equiv \frac{\left(\alpha s_{0}+\delta\right)^{2}+\gamma_{3}^{2}}{\beta^{2} \gamma_{3}}
$$

From here it follows that the process of resonant pumping is not essentially influenced by power broadening only during times smaller than $t_{c}$. After the time (82), the two-level resonant picture will not be adequate, since the 
neighboring nonresonant levels will be essentially involved in the process. The characteristic time (82), in view of Eq. $(77)$, is

$$
t_{c} \cong \frac{\left(\alpha s_{0}+\delta\right)^{2} \omega_{21}^{2}+\left(\alpha^{2}+\beta^{2}\right)^{2}}{\left(\alpha^{2}+\beta^{2}\right) \beta^{2} \omega_{21}} .
$$

Taking account of the inequalities $|\delta / \alpha| \ll 1$ and $|\beta / \alpha| \ll 1$, and setting $\left|s_{0}\right| \approx 1$, simplifies the time (83) to

$$
t_{c} \simeq \frac{\alpha^{2}+\omega_{21}^{2}}{\beta^{2} \omega_{21}}
$$

Finally, under conditions (46), Eq. (84) reduces to

$$
t_{c} \simeq \frac{\omega_{21}}{\beta^{2}}
$$

To estimate $t_{c}$ we may take $\beta \sim 0.1 \alpha$ and $\alpha \sim 0.1 \omega_{21}$, with a transition frequency, typical of magnetic traps [3], of order $\omega_{21} \sim 10^{2}-10^{3} \mathrm{~Hz}$. This gives $t_{c} \sim 10-100 \mathrm{~s}$, which is a rather long time, comparable or longer than the lifetime of atoms inside a trap $[2,3]$.

In this way, the temporal limitation resulting from the power broadening does not impose too severe restrictions on the procedure of the resonant excitation of coherent modes. Moreover, as is discussed in Sec. V, we do not need to pump too long but we may stop pumping as soon as the desired mode is populated, which happens during the time of order $2 \pi / \Omega$. The latter is of order $0.1 \mathrm{~s}$. Hence, the time necessary for populating a chosen coherent mode is essentially less than the critical time $t_{c}$, after which power broadening would spoil the resonant picture.

\section{INTERFERENCE PATTERNS}

In the process of the resonant excitation of coherent modes, only two modes are involved in the dynamical picture, while the population of all other nonresonant modes remains negligibly small. This allows us to present the system coherent function as the sum

$$
\varphi(\mathbf{r}, t)=\varphi_{1}(\mathbf{r}, t)+\varphi_{2}(\mathbf{r}, t)
$$

of the terms

$$
\varphi_{i}(\mathbf{r}, t)=c_{i}(t) \varphi_{i}(\mathbf{r}) \exp \left(-\frac{i}{\hbar} E_{i} t\right)
$$

corresponding to the related modes $i=1,2$. From here it follows that there should exist a spatial interference between the modes, similarly to the interference of different atomic components in a binary mixture of two Bose condensates [53]. In our case, the interference becomes possible because different coherent modes possess qualitatively different spatial shapes. Thus, we may define [3] the interference pattern

$$
\rho_{\text {int }}(\mathbf{r}, t) \equiv \rho(\mathbf{r}, t)-\rho_{1}(\mathbf{r}, t)-\rho_{2}(\mathbf{r}, t),
$$

in which

$$
\rho(\mathbf{r}, t) \equiv|\varphi(\mathbf{r}, t)|^{2}, \quad \rho_{i}(\mathbf{r}, t) \equiv\left|\varphi_{i}(\mathbf{r}, t)\right|^{2} .
$$

With the use of the dimensionless real function

$$
\psi_{i}(r, z) \equiv l_{r}^{3 / 2} \varphi_{i}(\mathbf{r}) e^{-i m_{i} \varphi},
$$

in which $l_{r}$ is the oscillator length and $m_{i}$ is a winding number, the pattern (88) reads

$$
\rho_{\text {int }}(\mathbf{r}, t)=\frac{\psi_{1} \psi_{2}}{l_{r}^{3}} \sqrt{1-s^{2}} \cos \Phi,
$$

where $s=s(t)$ is defined by Eq. (51) and the notation 


$$
\Phi \equiv\left(m_{2}-m_{1}\right) \varphi+x(t)-\gamma-\left(\omega_{21}+\Delta \omega\right) t
$$

is introduced. This interference pattern can be experimentally observed either inside the trap by means of light scattering or by freeing atoms from the trap and observing their free evolution, as is discussed by Sinatra and Castin [53].

The existence of two modes in a trap, with different spatial shapes of the related wave functions, leads to the appearance of atomic current inside the trap. This effect is analogous to the Josephson effect, which is usually considered for two atomic clouds sitting in a double-well potential, so that the clouds are separated by a potential barrier [29-32]. However, as is suggested by Leggett [54], Josephson oscillations can exist between two interpenetrating populations, not separated by any barrier. In this case, one calls it the internal Josephson effect [55]. Such a tunneling, involving no potential barriers, is also called quantum dynamical tunneling [56,57], which is actually just a current between two modes representing bound states.

The interference current is

$$
\mathbf{j}_{\text {int }}(\mathbf{r}, t) \equiv \mathbf{j}(\mathbf{r}, t)-\mathbf{j}_{1}(\mathbf{r}, t)-\mathbf{j}_{2}(\mathbf{r}, t)
$$

where

$$
\mathbf{j}(\mathbf{r}, t)=\frac{\hbar}{m_{0}} \operatorname{Im} \varphi^{*}(\mathbf{r}, t) \nabla^{2} \varphi(\mathbf{r}, t), \quad \mathbf{j}_{i}(\mathbf{r}, t)=\frac{\hbar}{m_{0}} \operatorname{Im} \varphi_{i}^{*}(\mathbf{r}, t) \nabla \varphi_{i}(\mathbf{r}, t),
$$

with $i=1,2$. Employing the notation in Eqs. (90) and (92), we find

$$
\mathbf{j}_{i n t}(\mathbf{r}, t)=\frac{\hbar \psi_{1} \psi_{2}}{2 m_{0} l_{r}^{4}} \sqrt{1-s^{2}}\left[\left(\mathbf{e}_{r} \frac{\partial}{\partial r}+\mathbf{e}_{z} \frac{\partial}{\partial z}\right) \ln \left(\frac{\psi_{2}}{\psi_{1}}\right) \sin \Phi+\mathbf{e}_{\varphi} \frac{m_{1}+m_{2}}{r} \cos \Phi\right] .
$$

Both the interference patterns (91) and interference current (95) experience temporal oscillations on two time scales. One corresponding to the transition frequency $\omega_{21}$, which is typical of the Josephson effect. And another scale is related to the temporal variation of $s(t)$ and $x(t)$, which is conneted with the transition amplitudes $\alpha$ and $\beta$. Since $\alpha, \beta \ll \omega_{21}$, the Josephson oscillations are fast, as compared to the slow change of $s(t)$ and $x(t)$. The fast Josephson oscillations, being modulated by the slow variation of $s(t)$ and $x(t)$, yield the collapse-revival picture typical of some two-level systems [26]. Here the modulation is mainly due to the temporal variation of $s(t)$. If one stops applying the resonant field, so that $\beta=0$, then $s(t)=$ const, and the slow modulation disappears.

\section{SPIN SQUEEZING}

The considered atomic system is essentially nonlinear. Therefore, one might expect that some kind of squeezing effects could arise. Similarly to squeezed states of light [58,59], one can introduce squeezed atomic states [58]. This is usually considered for the case of two-level atoms, each of which possesses two internal states. Finite level systems, as is known, are conveniently described by means of spin operators, because of which atomic squeezing is commonly called spin squeezing. In order to emphasize that the spin operators, employed for describing finite-level atoms, are not actually the operators representing real spins, but rather are convenient mathematical tools, one also uses the terms of dipole squeezing [58,60] or pseudospin squeezing [61]. In general, one may define squeezing for other operators from a Lie algebra [61,62]. Atomic squeezing is directly related to the radiation field squeezing [63]. And vice versa, squeezed atomic states can be created by irradiating atoms with squeezed light [64-67] or with light combined with an alternating magnetic field, as in the process of continuos quantum measurement [68]. Generally, atomic squeezing can be achieved for both bosons as well as for fermions [69,70]. Squeezing in Bose-Einstein condensates was considered for two-component mixtures [71], for atoms with two internal states [72], which is equivalent to a two-component mixture, and for atoms in linked mesoscopic traps formed by an optical lattice [73], which is equivalent to a multicomponent mixture. There is a real potential for several practical applications of squeezed atoms, e.g., for atomic spectroscopy and atomic clocks [74], for atom interferometers [75], and, probably, for quantum computation [76].

To consider spin squeezing in our case, we may notice that the transition dipole (71) and population difference (51) can be presented as the statistical averages

$$
h \equiv \frac{2}{N}<S_{-}>, \quad s \equiv \frac{2}{n}<S_{z}>
$$

of the collective spin operators 


$$
S_{\alpha} \equiv \sum_{i=1}^{N} S_{i}^{\alpha}, \quad S_{ \pm} \equiv S_{x} \pm i S_{y},
$$

where $\alpha=x, y, z$ and $S_{i}^{\alpha}$ corresponds to a $1 / 2$-spin operator. The evolution equations (73) and (74) can be obtained by averaging the Heisenberg equations of motion for the spin operators (97), with the effective Hamiltonian

$$
H_{\text {eff }}=\frac{\beta}{2}\left(S_{-}+S_{+}\right)-\delta S_{z}-\frac{\alpha}{N} S_{z}^{2} .
$$

Atomic squeezing is defined in a way similar to the squeezing of light, being based on the quantum-mechanical uncertainty relations. For any two operators $A$ and $B$, not necessarily Hermitian, the Heisenberg uncertainty relation reads

$$
\Delta^{2}(A) \Delta^{2}(B) \geq \frac{1}{4}|<[A, B]>|^{2},
$$

where the dispersion is

$$
\Delta^{2}(A) \equiv<A^{+} A>-|<A>|^{2} .
$$

One says that $A$ is squeezed with respect to $B$ if

$$
\Delta^{2}(A)<\frac{1}{2}|<[A, B]>| .
$$

This suggests to introduce the squeezing factor

$$
Q_{A B} \equiv \frac{2 \Delta^{2}(A)}{|<[A, B]>|} .
$$

Now, for $A$ and $B$, we may take any spin operators. However, taking separately $S_{\alpha}$, with $\alpha=x, y, z$, may involve the so-called trivial squeezing due to rotation [77]. To avoid this, we consider the operators $S_{z}$ and $S_{ \pm}$, for which we have

$$
\Delta^{2}\left(S_{z}\right) \Delta^{2}\left(S_{ \pm}\right) \geq \frac{1}{4}\left|<S_{ \pm}>\right|^{2} .
$$

Squeezing of $S_{z}$, with respect to $S_{ \pm}$, is defined by the squeezing factor $Q_{S_{z} S_{ \pm}} \equiv Q_{z}$, which in view of definition (101), is

$$
Q_{z}=\frac{2 \Delta^{2}\left(S_{z}\right)}{\left|<S_{ \pm}>\right|}
$$

The squeezing factor (103), taking into account that $\left|<S_{ \pm}>\right|^{2}=<S_{x}>^{2}+<S_{y}>^{2}$, becomes

$$
Q_{z}=\frac{2 \Delta^{2}\left(S_{z}\right)}{\sqrt{<S_{x}>^{2}+<S_{y}>^{2}}}
$$

which has the form used in Refs. [72,74,77]. One can say that $S_{z}$ is squeezed with respect to $S_{ \pm}$if $\Delta^{2}\left(S_{z}\right)<\frac{1}{2} \mid<$ $S_{ \pm}>\mid$, that is, $Q_{z}<1$. Calculating the expressions

$$
\Delta^{2}\left(S_{z}\right)=\frac{N}{4}\left(1-s^{2}\right), \quad \Delta^{2}\left(S_{ \pm}\right)=\frac{N}{4}\left(1 \mp s^{2}\right), \quad\left|<S_{ \pm}>\right|=\frac{N}{2}|h|=\frac{N}{2} \sqrt{1-s^{2}},
$$

we obtain the squeezing factor

$$
Q_{z}=\sqrt{1-s^{2}}
$$

where $s=s(t)$ is the population difference satisfying the evolution equations discussed above. As is seen, since $0 \leq s^{2} \leq 1$, the factor (105) is almost always less than one, except for $s=0$. Hence $S_{z}$ is squeezed with respect to $S_{ \pm}$. In other words, the dispersion $\Delta^{2}\left(S_{z}\right)$ is almost always smaller than the dispersion $\Delta^{2}\left(S_{ \pm}\right)$. This means that the population difference and, respectively, the fractional populations can be measured with a better accuracy than the 
transition dipole or the relative phase between population amplitudes. The characteristic temporal behaviour of the squeezing factor (105) is shown in Fig. 4, with $s(t)$ found numerically from the evolution equations.

It is worth noting that the treatment of atomic squeezing by means of spin operators is often used in the quantum optics language [58-61]. This is equivalent to the formation of squeezed states generated by the Bogolubov canonical transformation for quasiparticles in multicomponent (spinor) condensate. These two pictures are equivalent mathematically and are caused by the same physical reason, by the existence of nonlinear interactions in a multicomponent system.

In the theory of nuclear magnetic resonance [78,79], one distinguishes the state coherence, when $s= \pm 1$, and the transition coherence, when $|h|=1$. This terminology can also be applied to our case. Since here we have $|h|^{2}=1-s^{2}$, then the state and transition coherences are complimentary to each other. And if $Q_{z}<1$, this means that the state coherence can be better controlled than the transition coherence.

Atomic squeezing is usually neighbours with atomic entanglement $[72,80,81]$. Entanglement is simply the Schrödinger's name for superposition in a multiparticle system. In the system of trapped atoms or ions with two or more internal states, multiparticle entanglement can appear [81]. This concerns as well Bose-Einstein condensates composed of atoms having internal states $[72,82]$. The Bose-condensed atomic cloud, subject to the coherent resonance we study here, is also in a multiparticle entangled state. Really, the multiparticle density matrix, for the case studied, is

$$
\rho_{N}\left(\mathbf{r}_{1}, \mathbf{r}_{2}, \ldots, \mathbf{r}_{N}, \mathbf{r}_{1}^{\prime}, \ldots, \mathbf{r}_{N}^{\prime}, t\right)=\left|c_{1}(t)\right|^{2} \prod_{i=1}^{N} \varphi_{1}\left(\mathbf{r}_{i}\right) \varphi_{1}^{*}\left(\mathbf{r}_{i}^{\prime}\right)+\left|c_{2}(t)\right|^{2} \prod_{i=1}^{N} \varphi_{2}\left(\mathbf{r}_{i}\right) \varphi_{2}^{*}\left(\mathbf{r}_{i}^{\prime}\right),
$$

where $\left|c_{1}\right|^{2}+\left|c_{2}\right|^{2}=1$. This function cannot be written in any way as a product of single-particle functions. Hence $\rho_{N}$ is an entangled state, being a kind of the mixed counterpart of the Greenberger-Horne-Zeilinger state [83,84].

The principal difference of the two-mode coherent system, we consider, from the entanglement of trapped atoms, studied earlier, is that the nonlinear coherent modes describe not internal states of separate atoms, but collective coherent states of the whole system. Therefore this type of entanglement can be called coherent entanglement.

\section{RELAXATION PROCESS}

In the previous sections, we have considered the situation when Bose-condensed atoms are subject to the permanent pumping by an alternating resonant field. One might ask the question, what happens if this resonant pumping is stopped? How to describe the behaviour of the system after the coherent resonance?

If the resonant field ceases acting on the system, say at the moment of time $t_{0}$, then, after this time, the two-mode picture becomes invalid and one needs to return back to the initial equation (6) for the coherent field. This equation is to be complemented by the relaxation terms characterizing atomic collisions and possible existence of noncondensed atoms [1-3]. Then the coherent-field equation reads

$$
i \hbar \frac{\partial \eta}{\partial t}=H[\eta] \eta-\frac{i \hbar}{2}\left(K_{2}|\eta|^{2}+K_{3}|\eta|^{4}+\Gamma M\right) \eta,
$$

in which $H[\eta]$ is the nonlinear Hamiltonian (7), $K_{2}$ and $K_{3}$ are two-body and three-body recombination loss-rate coefficients [85], $\Gamma$ is the loss rate due to the transfer of condensed atoms to noncondensed atoms whose number is $M$. If a cooling mechanism is supported, so that there is an opposite process of transferring atoms from a noncondensed cloud to the condensate, then $\Gamma<0$ is a gain rate. The number of particles in the condensate is $N=\|\eta\|^{2}$. The initial condition for Eq. (106), in view of relation (8), is prescribed by the function

$$
\eta\left(\mathbf{r}, t_{0}\right)=\sqrt{N} \varphi\left(\mathbf{r}, t_{0}\right),
$$

with $\varphi(\mathbf{r}, t)$ given by the sum (86) taken at the moment $t_{0}$, when the resonant field stopped acting on the system.

In addition to loss rates caused by depolarizing collisions, there can exist another internal natural loss rate

$$
\Gamma_{1} \equiv-\frac{2}{\hbar} \operatorname{Im}(\varphi, \hat{H}[\varphi] \varphi)
$$

appearing for atoms with attractive interactions, if the number of atoms is larger than the critical number $N_{c}$. Here $\hat{H}[\varphi]$ is the nonlinear Hamiltonian (11). Thus, for a spherical trap, the loss rate (108) is estimated [4] as

$$
\Gamma_{1} \simeq 2.867 \omega_{r} \Theta\left(N-N_{c}\right)\left|\frac{a_{s}}{l_{r}} N\right|^{2 / 5},
$$


where $N \gg N_{c}$ and $\Theta(\cdot)$ is a unit step function. The loss rates due to two-body and three-body depolarizing collisions are

$$
\Gamma_{2} \equiv K_{2}\left(|\varphi|^{2},|\varphi|^{2}\right), \quad \Gamma_{3} \equiv K_{3}\left(|\varphi|^{3},|\varphi|^{3}\right) .
$$

From the evolution equation (106), we can easily derive the rate equation for condensed atoms, which, being complimented by the rate equation for noncondensed atoms, makes the general set of equations

$$
\frac{d N}{d t}=-\Gamma_{1} N-\Gamma_{2} N^{2}-\Gamma_{3} N^{3}-\Gamma M N, \quad \frac{d M}{d t}=-\gamma_{1}\left(M-M_{0}\right)+\Gamma N M,
$$

defining the relaxation process in the system after the coherent resonance. Here $\gamma_{1}$ is a pumping rate for noncondensed atoms and $M_{0}$ is their stationary number.

It is not our aim to give in this paper a detailed analysis of the relaxation equations (110). This is a separate problem which could be investigated in other publications. Here our main goal has been to present a thorough description of trapped Bose-Einstein condensate under the condition of coherent resonance. Therefore, we limit this section by a brief sketch of the way that would allow one to describe in detail what happens after the coherent resonance. The relaxation procedure defined by Eqs. (110) can follow quite different patterns depending on the concrete physical situation and on the values of the related relaxation rates. In a particular case, when the natural loss rate (108) is zero and the are no noncondensed atoms, the relaxation is completely due to depolarizing collisions. Then the relaxation time is the lifetime of atoms in a trap. For different traps this time varies between $10 \mathrm{~s}$ and $100 \mathrm{~s}$. Note that the loss rate (108) is zero for atoms with repulsive interactions and also for atoms with attractive interactions, if the number of atoms is less than critical. In such cases, nonlinear coherent modes, created by means of coherent resonance, can live, after the pumping resonance field is switched off, quite long time, of the order of the lifetime of atoms in a trap. This makes it feasible to study their behaviour as well as to use them for practical applications.

\section{CONCLUSIONS}

We have demonstrated that nonlinear coherent modes of trapped Bose-condensed atoms can be created by means of a resonant alternating field. These modes represent collective states corresponding to nonground-state Bose-Einstein condensates. Conditions, when such a resonant excitation of coherent modes is possible, are investigated. One restriction is that the number of atoms in a mode be less than a limiting number. The latter depends on the type of atoms and trap characteristics, and can be as large as $10^{5}$. This limiting number is close to that required for the stability of atoms with negative scattering lengths. Hence, atoms with attractive interactions can also be employed for creating nonlinear coherent modes. Moreover, since the mode limiting number increases for higher modes, it could be possible to stabilize a larger number of atoms with negative scattering length by transferring them to such excited coherent modes.

Another restriction on the coherent resonance is that the resonant pumping can last not longer than a critical time, before power broadening spoils the resonant picture. However, this limitation is not dangerous because of two reasons. First, the critical time for power broadening is rather long, of order $10-100 \mathrm{~s}$, which is about the lifetime of atoms in a trap. Second, there is no need to pump the system for so long times, since the transfer to an excited coherent mode takes essentially shorter times, around $0.1 \mathrm{~s}$.

Temporal behaviour of fractional populations, in the process of coherent resonance, exhibits dynamic critical phenomena occurring on a critical line in the parametric manifold. The related time-averaged system displays, on this critical line, critical effects typical of phase transitions. The origin of these critical phenomena is the saddle separatrix crossing by a starting point of a trajectory.

Interference patterns and interference current can be observed. These are related to the internal Josephson effect and to dynamic barrierless tunneling.

Atomic squeezing is realized demonstrating that the state coherence is better defined than transition coherence. Massive multiparticle entanglement is produced. A specific feature of this coherent entanglement, distinguishing it from earlier studies, is that it involves not internal states of individual atoms but collective coherent states of atomic condensate.

After the pumping resonant field is switched off, the behaviour of the system can be described by the rate equations. This behaviour depends on the physical setup defining the related relaxation rates. When the loss of atoms in a nonlinear coherent mode is caused by depolarizing atomic collisions, the lifetime of the mode is about $10-100 \mathrm{~s}$, which is the lifetime of atoms in a trap.

The two-mode picture of a resonant Bose condensate somewhat resembles the two-level picture of a resonant atom. However there is a principal difference between these two cases in the nature of the states involved. The notion of a 
resonant atom involves internal states of a single atom, while that of a resonant condensate has to do with collective coherent states of the whole system. Thus, the resonant condensate is a principally new resonant physical system. Its similarity with resonant atoms makes it feasible to extend to this type of systems various applications that are elaborated for resonant atoms. And its difference from the latter brings hopes of discovering new effects and finding novel applications.

\section{Acknowledgement}

One of the authors (V. I. Y.) is very grateful for discussions and useful comments to M. D. Girardeau, V. K. Melnikov, and E. Zaremba.

The work has been accomplished in the Research Center for Optics and Photonics, University of São Paulo, São Carlos. Financial support from the São Paulo State Research Foundation (Fapesp) is appreciated. 


\section{Appendix}

To illustrate the calculations of Sec. IV, we present here the related explicit expressions for several first nonlinear coherent modes, including the ground state mode $(n=m=j=0 ; p=q=1)$, radial dipole mode $(n=1, m=$ $j=0 ; p=3, q=1)$, basic vortex mode $(n=0, m=1, j=0 ; p=2, q=1)$, and axial dipole mode $(n=m=0, j=1 ; p=1, q=3)$. The corresponding wave functions are

$$
\begin{gathered}
\psi_{0}(r, \varphi, z)=\left(\frac{u_{0}^{2} v_{0}}{\pi^{3}}\right)^{1 / 4} \exp \left\{-\frac{1}{2}\left(u_{0} r^{2}+v_{0} z^{2}\right)\right\}, \\
\psi_{100}(r, \varphi, z)=\left(\frac{u_{100}^{2} v_{100}}{\pi^{3}}\right)^{1 / 4}\left(u_{100} r^{2}-1\right) \exp \left\{-\frac{1}{2}\left(u_{100} r^{2}+v_{100} z^{2}\right)\right\}, \\
\psi_{010}(r, \varphi, z)=u_{010}\left(\frac{v_{010}}{\pi^{3}}\right)^{1 / 4} r e^{i \varphi} \exp \left\{-\frac{1}{2}\left(u_{010} r^{2}+v_{010} z^{2}\right)\right\}, \\
\psi_{001}(r, \varphi, z)=\left(\frac{4 u_{001}^{2} v_{001}^{3}}{\pi^{3}}\right)^{1 / 4} z \exp \left\{-\frac{1}{2}\left(u_{001} r^{2}+v_{001} z^{2}\right)\right\},
\end{gathered}
$$

where $u_{n m j}$ and $v_{n m j}$ are the control functions defined by Eq. (39).

The corresponding integrals $I_{n m j}$ are

$$
I_{000}=(2 \pi)^{-3 / 2} \equiv I_{0}=0.063494, \quad I_{100}=\frac{1}{2} I_{0}, \quad I_{010}=\frac{1}{2} I_{0}, \quad I_{001}=\frac{3}{4} I_{0} .
$$

And the integrals (44) are

$$
\begin{gathered}
J_{100}=\frac{u_{100}\left(u_{0}^{2}+u_{100}^{2}\right)}{\pi^{3 / 2}\left(u_{0}+u_{100}\right)^{3}}\left(\frac{v_{100}}{v_{0}+v_{100}}\right)^{1 / 2}, \quad J_{010}=\frac{u_{010}^{2}}{\pi^{3 / 2}\left(u_{0}+u_{010}\right)^{2}}\left(\frac{v_{010}}{v_{0}+v_{010}}\right)^{1 / 2}, \\
J_{001}=\frac{u_{001}}{\pi^{3 / 2}\left(u_{0}+u_{001}\right)}\left(\frac{v_{001}}{v_{0}+v_{001}}\right)^{3 / 2} .
\end{gathered}
$$

In the weak-coupling limits, when $g \sqrt{\nu} \rightarrow 0$, the control functions behave as

$$
\begin{array}{rlrl}
u_{0} & \simeq 1-I_{0} g \sqrt{\nu}, & v_{0} & \simeq \nu-I_{0} g \sqrt{\nu}, \\
u_{100} \simeq 1-\frac{1}{6} I_{0} g \sqrt{\nu}, & v_{100} & \simeq \nu-\frac{1}{2} I_{0} g \sqrt{\nu}, \\
u_{010} \simeq 1-\frac{1}{4} I_{0} g \sqrt{\nu}, & v_{010} & \simeq \nu-\frac{1}{2} I_{0} g \sqrt{\nu}, \\
u_{001} & \simeq 1-\frac{3}{4} I_{0} g \sqrt{\nu}, & v_{001} & \simeq \nu-\frac{1}{4} I_{0} g \sqrt{\nu} .
\end{array}
$$

And for the energy spectrum (42), we have

$$
\begin{aligned}
E_{0} & \simeq 1+\frac{\nu}{2}+I_{0} g \sqrt{\nu}, & E_{100} & \simeq 3+\frac{\nu}{2}+\frac{1}{2} I_{0} g \sqrt{\nu}, \\
E_{010} & \simeq 2+\frac{\nu}{2}+\frac{1}{2} I_{0} g \sqrt{\nu}, & E_{001} & \simeq 1+\frac{3 \nu}{2}+\frac{3}{4} I_{0} g \sqrt{\nu} .
\end{aligned}
$$


Then the transition frequency (16), for a transition between the ground state and an excited mode, becomes, respectively,

$$
\omega_{100,0} \simeq 2-0.031747 g \sqrt{\nu}, \quad \omega_{010,0} \simeq 1-0.031747 g \sqrt{\nu}, \quad \omega_{001,0} \simeq \nu-0.015874 g \sqrt{\nu}
$$

And the transition amplitudes (45) take the values

$$
\begin{gathered}
\alpha_{0,100} \simeq \alpha_{0,010} \simeq \alpha_{0,001} \simeq 0 \\
\alpha_{100,0} \simeq \alpha_{010,0} \simeq 0.031747 g \sqrt{\nu}, \quad \alpha_{001,0} \simeq 0.015874 g \sqrt{\nu} .
\end{gathered}
$$

Hence, in the weak-coupling limit, conditions (46) are always valid.

In the strong-coupling limit $g \nu \rightarrow \infty$, for the control functions we get

$$
\begin{array}{rlrl}
u_{0} & \simeq \frac{2.282947}{(g \nu)^{2 / 5}}, & v_{0} \simeq \frac{2.282947}{(g \nu)^{2 / 5}} \nu^{2}, \\
u_{100} \simeq \frac{5.823454}{(g \nu)^{2 / 5}}, & v_{100} \simeq \frac{1.941151}{(g \nu)^{2 / 5}} \nu^{2}, \\
u_{010} \simeq \frac{4.565895}{(g \nu)^{2 / 5}}, & v_{010} \simeq \frac{2.282947}{(g \nu)^{2 / 5}} \nu^{2}, \\
u_{001} \simeq \frac{2.056114}{(g \nu)^{2 / 5}}, & v_{001} \simeq \frac{6.168342}{(g \nu)^{2 / 5}} \nu^{2},
\end{array}
$$

which shows that in this limit the effective oscillator frequencies diminish. For the energies (43), we find

$$
\begin{aligned}
& E_{0} \simeq 0.547539(g \nu)^{2 / 5}+0.570736 \frac{2+\nu^{2}}{(g \nu)^{2 / 5}}, \quad E_{100} \simeq 0.643949(g \nu)^{2 / 5}+0.485287 \frac{18+\nu^{2}}{(g \nu)^{2 / 5}} \\
& E_{010} \simeq 0.547539(g \nu)^{2 / 5}+0.570736 \frac{8+\nu^{2}}{(g \nu)^{2 / 5}}, \quad E_{001} \simeq 0.607942(g \nu)^{2 / 5}+0.514029 \frac{2+9 \nu^{2}}{(g \nu)^{2 / 5}}
\end{aligned}
$$

Notice that passing from the weak-coupling to strong-coupling limit, the effect of level crossing happens, since the order of energy levels can be changed. Specifics of the level crossing depend on the value of the aspect ration $\nu$. For instance, if the trap is cigar-shape $(\nu \ll 1)$ then the arrangements of the energy levels in the weak-coupling limit,

$$
E_{0}<E_{001}<E_{010}<E_{100} \quad(g \rightarrow 0)
$$

changes, in the strong-coupling limit, to

$$
E_{0}<E_{010}<E_{001}<E_{100} \quad(g \rightarrow \infty) .
$$

The transition frequencies in the strong-coupling limit are

$$
\omega_{100,0} \simeq 0.096410(g \nu)^{2 / 5}, \quad \omega_{010,0} \simeq \frac{3.424416}{(g \nu)^{2 / 5}}, \quad \omega_{001,0} \simeq 0.060403(g \nu)^{2 / 5} .
$$

For the integral (44), we have

$$
J_{100} \simeq 0.052069, \quad J_{010}=0.056439, \quad J_{001} \simeq 0.053063 .
$$

Then the transition amplitudes (45) become

$$
\alpha_{0,100} \simeq 0.140202(g \nu)^{2 / 5}, \quad \alpha_{100,0} \simeq 0.101636(g \nu)^{2 / 5},
$$




$$
\begin{array}{ll}
\alpha_{0,010} \simeq 0.170345(g \nu)^{2 / 5}, & \alpha_{010,0} \simeq 0.170345(g \nu)^{2 / 5}, \\
\alpha_{0,001} \simeq 0.147059(g \nu)^{2 / 5}, & \alpha_{001,0} \simeq 0.122898(g \nu)^{2 / 5} .
\end{array}
$$

This shows that in the asymptotic limit $g \nu \rightarrow \infty$, inequalities (46) do not hold. They become valid outside the region of convergence of this asymptotic limit. The corresponding condition (48), for the considered lower modes, yields

$$
\begin{aligned}
& |g \nu| \leq 1.1\left(2+\nu^{2}\right)^{5 / 4} \quad(p=1, q=1), \\
& |g \nu| \leq 0.7\left(18+\nu^{2}\right)^{5 / 4} \quad(p=3, q=1), \\
& |g \nu| \leq 1.1\left(8+\nu^{2}\right)^{5 / 4} \quad(p=2, q=1), \\
& |g \nu| \leq 0.8\left(2+9 \nu^{2}\right)^{5 / 4} \quad(p=1, q=3) .
\end{aligned}
$$

These inequalities demonstrate that large values of the coupling parameter $g \gg 1$ can always be compensated by accepting an appropriate trap shape, being either cigar-shaped $(\nu \ll 1)$ or pancake-shaped $(\nu \gg 1)$.

[1] A. S. Parkins and D. F. Walls, Phys. Rep. 303, 1 (1998).

[2] F. Dalfovo, S. Giorgini, L. P. Pitaevskii, and S. Stringari, Rev. Mod. Phys. 71, 463 (1999).

[3] P.W. Courteille, V. S. Bagnato, and V. I. Yukalov, Laser Phys. 11, 659 (2001).

[4] V. I. Yukalov, E. P. Yukalova, and V. S. Bagnato, Phys. Rev. A 56, 4845 (1997).

[5] K. P. Marzlin and W. Zhang, Phys. Rev. A 57, 3801 (1998).

[6] K. P. Marzlin and W. Zhang, Phys. Rev. A 57, 4761 (1998).

[7] V. I. Yukalov, E. P. Yukalova, and V. S. Bagnato, Laser Phys. 10, 26 (2000).

[8] E. A. Ostrovskaya, Y. S. Kivshar, M. Lisak, B. Hall, F. Cattani, and D. Anderson, Phys. Rev. A 61, 031601 (2000).

[9] D. L. Feder, M. S. Pindzola, L. A. Collins, B. I. Scheider, and C. W. Clark, Phys. Rev. A 62, 053606 (2000).

[10] Y. S. Kivshar, T.J. Alexander, and S. K. Turitsyn, Phys. Lett. A 278, 225 (2001).

[11] B. Damski, Z. P. Karkuszewski, K. Sacha, and J. Zakrewski, Phys. Rev. A 56, 013604 (2001).

[12] R. D'Agosta, B. A. Malomed, and C. Precilla, Laser Phys. 12, 37 (2002).

[13] J. Williams, W. Walser, J. Cooper, E. A. Cornell, and M. Holland, Phys. Rev. A 61, 033612 (2000).

[14] V. I. Yukalov, Statistical Green's Functions (Queen's University, Kingston, 1998).

[15] F. Calogero and A. Degasperis, Spectral Transform and Solitons (North-Holland, Amsterdam, 1982).

[16] L. D. Faddeev and L. A. Takhtajan, Hamiltonian Methods in the Theory of Solitons (Springer, Berlin, 1987).

[17] C. Sulem and P. L. Sulem, Nonlinear Schrödinger Equation: Self Focusing and Wave Collapse (Springer, New York, 1999).

[18] Y. S. Kivshar and B. A. Malomed, Rev. Mod. Phys. 61, 763 (1989).

[19] V. I. Yukalov, E. P. Yukalova, and V. S. Bagnato, Laser Phys. 11, 455 (2001).

[20] V. I. Yukalov, E. P. Yukalova, and V. S. Bagnato, Laser Phys. 12, 231 (2002).

[21] J. R. Klauder and B. S. Skagerstam, Coherent States (World Scientific, Singapore, 1985).

[22] Encyclopedia of Mathematics, edited by I. M. Vinogradov (Kluwer, Dordrecht, 1988), Vol. 2, p. 67.

[23] P. E. Zhidkov, Int. J. Mod. Phys. A 12, 295 (1997).

[24] P. E. Zhidkov, Nonlinear Anal. 43, 471 (2001).

[25] K. Goral, M. Gajda, and K. Rzazewski, Opt. Express. 8, 92 (2001).

[26] L. Allen and J. H. Eberly, Optical Resonance and Two-Level Atoms (Wiley, New York, 1975).

[27] N. N. Bogolubov and Y. A. Mitropolsky, Asymptotic Methods in the Theory of Nonlinear Oscillations (Gordon and Breach, New York, 1961).

[28] L. Mandel and E. Wolf, Optical Coherence and Quantum Optics (Cambridge University, Cambridge, 1995).

[29] F. Dalfovo, L. P. Pitaevskii, and S. Stringari, Phys. Rev. A 54, 4213 (1996).

[30] A. Smerzi, S. Fantoni, S. Giovanazzi, and S. R. Shenoy, Phys. Rev. Lett. 79, 4950 (1997).

[31] G. J. Milburn, J. Corney, E.M. Wright, and D. F. Walls, Phys. Rev. A 55, 4318 (1997).

[32] S. Raghhavan, A. Smeri, S. Fantoni, and S. R. Shenoy, Phys. Rev. A 59, 620 (1999). 
[33] V. I. Yukalov, Mosc. Univ. Phys. Bull. 31, 10 (1976).

[34] V. I. Yukalov, Theor. Math. Phys. 28, 652 (1976).

[35] V. I. Yukalov, Ann. Physik 38, 419 (1981).

[36] I. V. Dobrovolska and R. S. Tutik, Int. J. Mod. Phys. A 16, 2493 (2001).

[37] C. A. Sackett, C. C. Bradley, M. Welling, and R. G. Hulet, Appl. Phys. B 65, 433 (1997).

[38] J. L. Roberts, N. R. Claussen, S. L. Cornish, E. A. Donley, E. A. Cornell, and C. E. Wieman, Phys. Rev. Lett. 86, 4211 (2001).

[39] P. A. Ruprecht, M. J. Holland, K. Burnett, and M. Edwards, Phys. Rev. A 51, 4704 (1995).

[40] M. Ueda and K. Huang, Phys. Rev. A 60, 3317 (1999).

[41] A. Gammal, T. Frederico, and L. Tomio, Phys. Rev. A 64, 055602 (2001).

[42] Y. Kagan, G. V. Shlyapnikov, and J. T. M. Walraven, Phys. Rev. Lett. 76, 2670 (1996).

[43] H. T. C. Stoof, J. Stat. Phys. 87, 1353 (1997).

[44] M. Ueda and A. J. Leggett, Phys. Rev. Lett. 80, 1576 (1998).

[45] A. K. Pattanayak, A. Gammal, C. A. Sackett, and R. G. Hulet, Phys. Rev. A 63, 033604 (2001).

[46] M. R. Andrews et. al., Science 275, 637 (1997).

[47] R. Franzosi and V. Penna, Laser Phys. 12, 71 (2002).

[48] C. W. Gardiner, Handbook of Stochastic Methods (Springer, Berlin, 1997).

[49] V. I. Yukalov, Phys. Rev. Lett. 75, 3000 (1995).

[50] V. I. Yukalov, Laser Phys. 5, 970 (1995).

[51] V. I. Yukalov, Phys. Rev. B 53, 9232 (1996).

[52] J. Sanders and F. Verhulst, Averaging Methods in Nonlinear Dynamical Systems (Springer, New York, 1985).

[53] A. Sinatra and Y. Castin, Eur. Phys. J. D 8, 319 (2000).

[54] A. J. Leggett, Rev. Mod. Phys. 47, 331 (1975).

[55] P. Ohberg and S. Stenholm, Phys. Rev. A 59, 3890 (1999).

[56] M. J. Davis and E. J. Heller, J. Chem. Phys. 75, 246 (1981).

[57] E. J. Heller and M. J. Davis, J. Phys. Chem. 85, 307 (1981).

[58] R. Loudon and P. L. Knight, J. Mod. Opt. 34, 709 (1987).

[59] H. J. Carmichael, A. S. Lane, and D. F. Walls, J. Mod. Opt. 34, 821 (1987).

[60] S. M. Barnett and M. A. Dupertuis, J. Opt. Soc. Am. B 4, 505 (1987).

[61] P. K. Aravind, J. Opt. Soc. Am. B 5, 1545 (1988).

[62] K. Wodkiewicz and J. H. Eberly, J. Opt. Soc. Am. B 2, 458 (1985).

[63] D. F. Walls and P. Zoller, Phys. Rev. Lett. 47, 709 (1981).

[64] G. S. Agarwal and R. R. Puri, Phys. Rev. A 41, 3782 (1990).

[65] A. Kuzmich, K. Mølmer, and E. S. Polzik, Phys. Rev. Lett. 79, 4782 (1997).

[66] K. Mølmer, Eur. Phys. J. D 5, 301 (1999).

[67] J. Hald, J. L. Sørensen, C. Schori, and E. S. Polzik, Phys. Rev. Lett. 83, 1319 (1999).

[68] A. Kuzmich, L. Mandel, and N. P. Bigelow, Phys. Rev. Lett. 85, 1594 (2000).

[69] B. Yurke, Phys. Rev. Lett. 56, 1515 (1986).

[70] K. Svozil, Phys. Rev. Lett. 65, 3341 (1990).

[71] U. V. Poulsen and K. Mølmer, Phys. Rev. A 64, 013616 (2001).

[72] A. Sørensen, L. M. Duan, J. I. Cirac, and P. Zoller, Nature 409, 63 (2001).

[73] C. Orzel, A. K. Tuchman, M. L. Fenselau, M. Yasuda, and M. A. Kasevich, Science 291, 2387 (2001).

[74] D. J. Wineland, J. J. Bollinger, W. M. Itano, and D. J. Heisen, Phys. Rev. A 50, 67 (1994).

[75] P. Boyer and M. A. Kasevich, Phys. Rev. A 56, 1083 (1997).

[76] A. Sørensen and K. Mølmer, Phys. Rev. Lett. 82, 1971 (1999).

[77] M. Kitagawa and M. Ueda, Phys. Rev. A 47, 5138 (1993).

[78] C. P. Slichter, Principles of Magnetic Resonance (Springer, Berlin, 1978).

[79] R. R. Ernst, G. Bodenhausen, and A. Wokaun, Principles of Nuclear Resonance in One and Two Dimensions (Clarendon, Oxford, 1987).

[80] C. H. Bennet, D. P. DiVincenzo, J. A. Smolin, and W. K. Wooters, Phys. Rev. A 54, 3824 (1996).

[81] K. Mølmer and A. Sørensen, Phys. Rev. Lett. 82, 1835 (1999).

[82] K. Helmerson and L. You, Phys. Rev. Lett 87, 170402 (2001).

[83] D. M. Greenberger, M. A. Horne, A. Shimony, and A. Zeilinger, Am. J. Phys. 58, 1131 (1990).

[84] D. M. Greenberger, M. A. Horne, and A. Zeilinger, Phys. Today 46, N 8, 22 (1993).

[85] J. Weiner, V. S. Bagnato, S. Zilio, and P. S. Juliene, Rev. Mod. Phys. 71, 1 (1999). 


\section{Figure Captions}

Fig. 1. Characteristic evolution of the phase portrait on the population difference, $s(t)$, - phase difference, $x(t)$, plane, under zero detuning $\varepsilon=0$ and varying amplitude of the resonant field: (a) $b=0.1$, (b) $b=0.4$; (c) $b=0.49$; (d) $b=0.51 ;$ (e) $b=0.8$.

Fig. 2. Dramatic change in the temporal behaviour of the population difference $s(t)$ (dashed line) and phase difference $x(t)$ (solid line) when crossing the critical line at the point $\left\{b_{c}=0.5, \varepsilon=0\right\}$. The initial conditions are $s_{0}=-1, x_{0}=0$. Time is presented in dimensionless units, as explained in the text. In Figs. 2(a) and 2(b), the value of $b$ is, respectively, just below and just above the critical point $b_{c}$, with the variation of $\Delta b=10^{-5}$.

Fig. 3. The change of dynamics in the population difference $s(t)$ (dashed line) and phase difference $x(t)$ (solid line) when crossing the critical line at the point $\left\{b_{c}=-0.5, \varepsilon=0\right\}$. The initial conditions are $s_{0}=-1, x_{0}=0$. Time is dimensionless (see the text). In Figs. 3(a) and 3(b), the value of $b$ is, respectively, slightly above and slightly below the critical point $b_{c}$, with the variation $\Delta b \sim 10^{-5}$.

Fig. 4. The squeezing factor $Q_{z}$ as a function of time for the transition amplitude $b=0.49$, zero detuning, and the initial conditions $s_{0}=-1$ and $x_{0}=0$. 